\title{
How stable are our standard candles? Time-resolved analysis of amplitude and period changes in OGLE-III LMC Cepheids
}

\author{
Maria Süveges ${ }^{1}$, Richard I. Anderson ${ }^{2}$ and Laurent Eyer ${ }^{1}$ \\ ${ }^{1}$ Dept. of Astronomy, University of Geneva, Switzerland \\ email: Maria.Suveges@unige.ch, Laurent.Eyer@unige.ch \\ ${ }^{2}$ Dept. of Physics and Astronomy, Johns Hopkins University, Baltimore, MD, USA \\ email: ria@jhu.edu
}

\begin{abstract}
We revisit a group of OGLE-III Cepheids that exhibit nearby double peaks in their periodograms using a technique of statistics called kernel modelling. We investigate the phenomenology of these Cepheids (termed twin-peak Cepheids in this paper) in more detail by comparing a sample of 29 LMC Cepheids that exhibit twin frequency peaks with 24 other Cepheids that do not. Using the kernel technique, we investigate light curve variability as a function of time, revealing both frequency and amplitude modulations. We present the preliminary results of our study in progress, which suggests a complex interplay between the two types of modulations and their detectability via twin peaks in the periodogram. The study reveals the potential of the kernel technique to help theory and modelling with detailed data analyses, capable of tracing fine time-dependent variations of the phenomenology of pulsations for objects of surveys or observation campaigns producing sufficiently dense time sampling.
\end{abstract}

Keywords. methods: data analysis, methods: statistical, stars: oscillations, Cepheids, Magellanic Clouds

\section{Introduction}

Although classical Cepheids have a reputation of being remarkably stable pulsating stars, it is becoming increasingly clear that a number of them are not perfectly repetitive: their amplitude, period or both can show variations (for photometric amplitude, see, e.g., Burki \& Mayor (1980); Molnár \& Szabados (2014); Percy \& Kim (2014); for radial velocity modulations, Burki \& Mayor (1980); Anderson (2014) and Anderson et al. (2015) in this volume; and for period variations, Szabados (1983); Poleski (2008); Derekas et al. (2012); Evans et al. (2015)).

In our study, we aimed to develop a statistical model which can decompose and quantify the phenomenology of these variations as a function of time, based on a Fourier formalism. The perspective is to apply the methods presented here on the large and temporally well-sampled population of OGLE-III Cepheids, in order to identify different types of variations and study their occurrence as a function of astrophysical parameters such as colour, pulsation mode or pulsation period.

\section{Sample}

We present our preliminary results obtained on the I-band observations of 53 classical Cepheids from the OGLE-III survey. Any instability of the pulsation parameters imply that a pre-whitening procedure with constant parameters fails to remove all variations from the light curve, so a peak in the residual periodogram close to the main pulsation 
period (termed in this paper a twin peak) can be the sign of varying parameters. We have thus selected 12 fundamental and 12 first overtone Cepheids showing a twin peak. 5 more first overtone Cepheids were added because of visible amplitude changes in their folded light curve. Finally, for comparison, 12 fundamental and 12 first-overtone stars with flat residual periodograms (i.e. with no clear signs of temporal changes in their pulsation) have also been included. The 29 objects showing twin peaks will be called twin-peak Cepheids, while the 24 stars with flat residual periodograms, control Cepheids.

\section{Methodology}

Let $Y_{i}$ denote the observed magnitudes at time $t_{i}$ with error bar $\sigma_{i}$. The fitted model is

$$
Y_{i}=\sum_{j=0}^{3} a_{j} t_{i}^{j}+\sum_{m=1}^{M}\left(s_{m} \sin 2 \pi m f t_{i}+c_{m} \cos 2 \pi m f t_{i}\right)+\epsilon_{i}
$$

with a polynomial term corresponding to a slow (instrumental or astrophysical) drift of the mean magnitude, a harmonic series describing the periodic light variation, and independent errors $\epsilon_{i} \sim \mathcal{N}\left(0, \sigma_{i}^{2}\right)$. We fit this model in 3-year long windows sliding over the total time span of the observations, obtaining different coefficients in the different windows. In order to obtain the most precise possible estimates at the window centre, we use a combined weighting scheme, multiplying the usual inverse squared error weights with a kernel that gives large weight to observations close to the window centre and small weight to those distant from the centre. Using window centres separated by 30 days (say at $\left.\tau_{1}, \ldots, \tau_{K}\right)$, and repeating optimization of model (3.1) in each window, we obtain the best local estimates : $a_{j}\left(\tau_{1}\right), \ldots, a_{j}\left(\tau_{K}\right) ; s_{m}\left(\tau_{1}\right), \ldots, s_{m}\left(\tau_{K}\right) ; c_{m}\left(\tau_{1}\right), \ldots, c_{m}\left(\tau_{K}\right)$ and $f\left(\tau_{1}\right), \ldots, f\left(\tau_{K}\right)$ for all $j$ and $m$. To obtain a conservative, prudent estimate of significance of the departure from stable estimates, we used sliding window estimates on a stationary model with bootstrapped residuals, and assessed significances by the multiple testing method of Benjamini \& Yekutieli (2001), accounting for dependency of the tests.

\section{Results}

Figure 1 presents a few examples of the variations found. The left column shows the temporal dependence of the pulsation period for four twin-peak Cepheids (upper four panels) and for one typical example of control Cepheid (lowermost panel). The range of the observed kinds of variations is very broad, from a near-linear trend to almost periodic-looking fluctuations to any combination of the two. The twin-peak and the control Cepheids do not seem to have distinct, easy-to-separate behaviours, but in the control group, these remain statistically non-significant according to our conservative residual-based bootstrap procedure, whereas in the twin-peak group, it is significant for the majority of the objects. The middle column shows typical variations of the first harmonic amplitude, $A_{1}=\left(s_{1}^{2}+c_{1}^{2}\right)^{1 / 2}$ (upper four panels: twin-peak Cepheids, bottom panel: control Cepheid). A subgroup of 8 overtone Cepheids seems to show strong significant fluctuations in this parameter, with changes up to 0.06 magnitude. Though fundamental mode stars also can show changes of this order, the size of the changes in their first harmonic amplitude remains on average smaller. The light curve shape (shown in the rightmost column for three first overtone stars in the top three panels and two fundamental stars in the bottom two panels) seem to be relatively more variable for the first overtone Cepheids than for the fundamental ones, though the absolute size of the changes is comparable. 

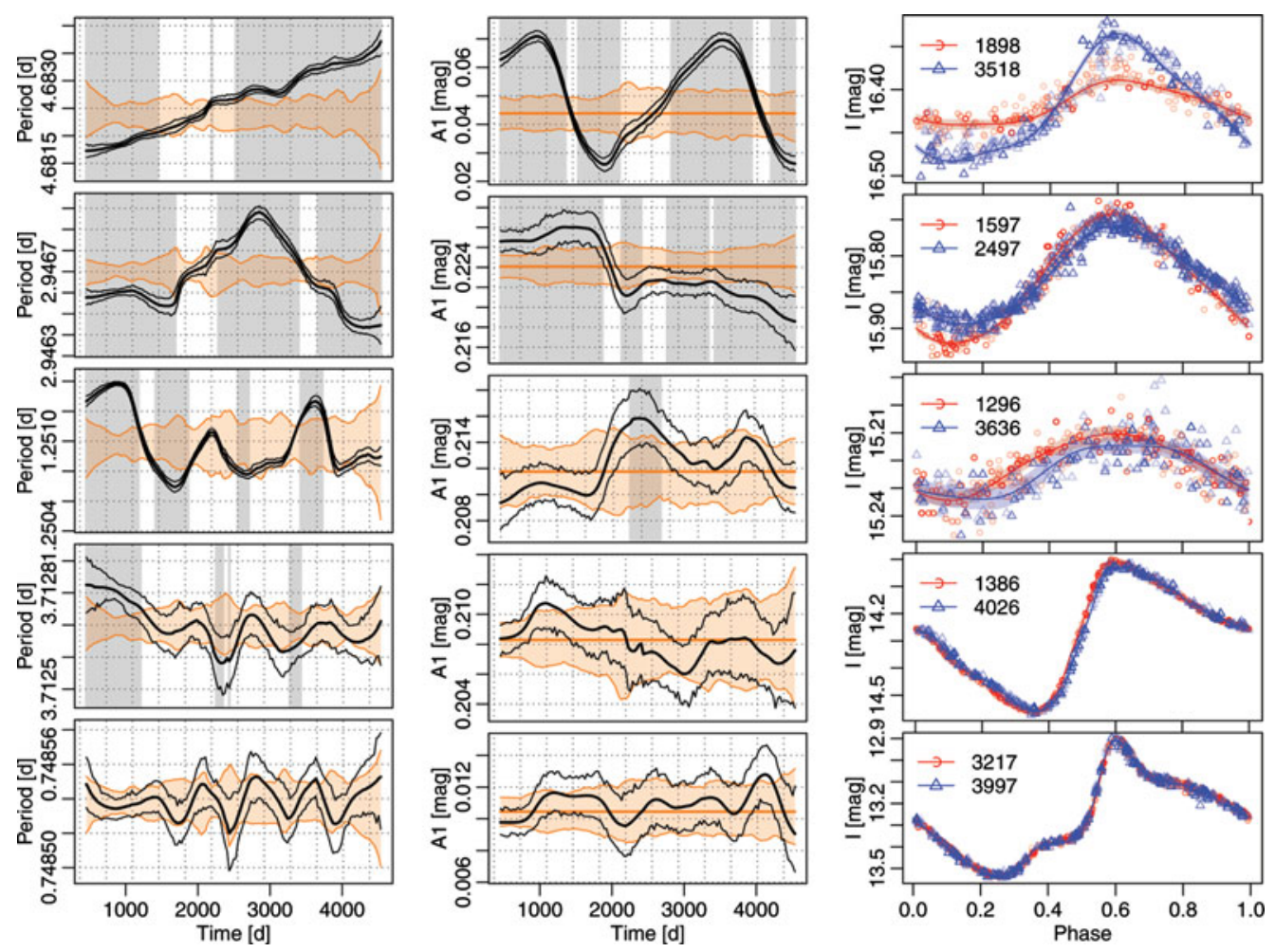

Figure 1. Examples of the time-dependent variations of the pulsation period (left column), the first harmonic amplitude $A_{1}$ (middle) and the light curve shape (right). In the left and middle columns, black lines indicate the variations estimated by the sliding window technique and its $95 \%$ confidence interval. The orange bands show the $95 \%$ confidence interval of a hypothetic stable Cepheid. The grey bands mark intervals of significant departures from this stable model. The times are given relative to JD 2450000. In the right column, red and blue show folded light curve shapes in the windows centred at the times indicated in the legend. Dark symbols correspond to observations close to the window centre, light ones to observations at the window ends.

In order to quantify these variations, we have fitted an approximate model consisting of a linear trend and periodic oscillations to the time series of estimated pulsation periods for each star, using an autoregressive model for strongly correlated errors. An example fit is shown in the leftmost panel of Figure 2, illustrating that though the fit is only a rough heuristic approximation, the general morphology of the variations is captured. The slope of the linear trend and the frequency and amplitude of the oscillatory term for different groups of Cepheids are compared in the other three panels of Figure 2. It seems that the presence of a twin peak in the residual periodogram indicates most often a global average trend (middle left) or a slow periodic component (middle right) in the variations. On the $\sim 12$ year time span of the OGLE-III observations, irregular trends and fluctuations of long periods ( $>12$ years) cannot be distinguished. In the rightmost panel of Figure 2, the ratio of the size of the period fluctuations to the average period of the Cepheid is shown. The differences between the fundamental and the first overtone groups seem to be more important than between the twin-peak and the control groups. Due to our selection procedure and the low number of Cepheids analysed, we cannot yet draw conclusions as to the statistical distribution of these parameters in the population of classical Cepheids. An application of the procedure on a larger sample, selected only 

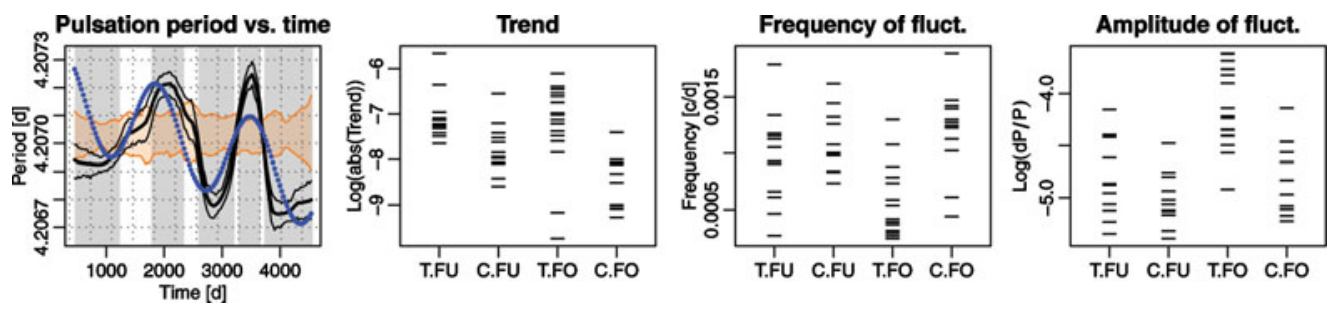

Figure 2. Phenomenology of the period fluctuations. Left panel: an example of a fit with linear and oscillatory terms for the period variations of a Cepheid. The blue dots show the fitted values, superposed to a plot similar to those in the left panel of Figure 1. The other three panels show the values of the fitted slope of the trend (middle left), the frequency of the oscillation (middle right), and the logarithm of $d P / P$ (the amplitude $d P$ of the oscillations of the pulsation period relative to the mean period $P$ of the star) for all 53 objects in the sample. T.FU: twin-peak fundamental-mode, T.FO: twin-peak first overtone, C.FU: control fundamental-mode, C.FO: control first overtone.

based on sufficient number of points instead of the aspect of their residual periodogram, is planned for the future.

\section{Summary}

Our study suggests that irregular variations of period and Fourier parameters can be a common phenomenon for Cepheids, with arbitrary combinations of changes on a wide range of timescales. Fluctuations seem to be ubiquitous; they can appear stochastic or quasi-periodic, similar to the radial velocity amplitude variations reported by Anderson (2014). Trends are less frequent, affecting mostly pulsation period; they can appear near-linear or irregular on the OGLE-III timespan. Cepheids with strong slow changes can be identified based on the presence of twin peaks in a frequency analysis of the residuals, since in these cases the pre-whitening with constant parameters is not able to sufficiently remove the main variability. Overtone pulsators appear somewhat less stable than fundamental ones, similar to the findings of Evans et al. (2015), though we find more instability among fundamental Cepheids. In particular, modulations of the first harmonic amplitude are more frequent and stronger among them, and the size of the changes in their pulsation period relative to their mean period is on average larger than those of fundamental Cepheids.

\section{References}

Anderson, R. I. 2014, $A \mathscr{E} A$, 566, id.L10

Anderson, R. I., Mérand, A., Kervella, P., Breitfelder, J., Eyer, L., \& Gallenne, A. 2015, IAU Meeting 2015, Honolulu, Hawaii, Focus Meeting 17, poster 09

Benjamini, Y. \& Yekutieli, D. 2001, Annals of Statistics, 29, 1165

Burki, G. \& Mayor, M. 1980, A\&A, 91, 115

Derekas, A., Szabó, Gy. M., Berdnikov, L., Szabó, R., Smolec, R., Kiss, L. L., Szabados, L., Chadid, M., Evans, N. R., Kinemuchi, K., Nemec, J. M., Seader, S. E., Smith, J. C., \& Tenenbaum, P. 2012, MNRAS, 425, 1312

Evans, N. R., Szabó, R., Derekas, A., Szabados, L., Cameron, C., Matthews, J. M., Sasselov, D., Kuschnig, R., Rowe, J. F., Guenther, D. B., Moffat, A. F. J., Rucinski, S. M., \& Weiss, W. W. 2015, MNRAS, 446, 4008

Molnár, L. \& Szabados, L. 2014, MNRAS, 422, 3222

Poleski, R. 2008, Acta Astronomica, 58, 313

Percy, J. R. \& Kim, R. Y-H. 2014, JAAVSO, 42, 1

Szabados, L. 1983, ApSS, 96, 185 